\title{
Efficiency and marginal cost pricing in dynamic competitive markets with friction
}

\author{
IN-Koо ChO \\ Department of Economics, University of Illinois \\ SEAN P. MEYN \\ Department of Electrical and Computer Engineering, University of Illinois
}

\begin{abstract}
This paper examines a dynamic general equilibrium model with supply friction. With or without friction, the competitive equilibrium is efficient. Without friction, the market price is completely determined by the marginal production cost. If friction is present, no matter how small, then the market price fluctuates between zero and the "choke-up" price, without any tendency to converge to the marginal production cost, exhibiting considerable volatility. The distribution of the gains from trading in an efficient allocation may be skewed in favor of the supplier, although every player in the market is a price taker.
\end{abstract}

KeYwords. Dynamic general equilibrium model with supply friction, choke-up price, marginal production cost, welfare theorems.

JEL CLASSIFICATION. D41, D51.

\section{INTRODUCTION}

The production of any commodity is subject to a number of constraints that are collectively known as friction. Examples abound. In a large organization such as a hospital or a call center, a large workforce must be maintained to ensure effective delivery of services. New employees are required to increase capacity of service, but proper talent must be identified and trained to be placed in position, which takes time. In supplying a seasonal fashion product, the retailer maintains a small inventory that is available at short notice, while maintaining a contract with the supplier for deliverables in case of an unexpected surge in demand. It is more economical to have such a contract than to maintain inventory, but it takes some time to deliver the product from the supplier to the retailer if demand increases unexpectedly (Dasgupta et al. 2005).

In-Koo Cho: inkoocho@uiuc.edu

Sean P. Meyn: meyn@control.csl.uiuc.edu

Numerous conversations with Ramesh Johari, Robert Thomas, and Robert Wilson, and critiques from the anonymous referees and the editor helped us improve the substance of the paper significantly. In particular, discussions with R. Johari led to improvements in the main result of this paper. Financial support from the National Science Foundation (ECS-0523620) and Deutsche Bank through the Institute for Advanced Study is gratefully acknowledged. Any opinions, findings, and conclusions or recommendations expressed in this material are those of the authors and do not necessarily reflect the views of the National Science Foundation, Deutsche Bank, or the Institute of Advanced Study.

Copyright ( 2010 In-Koo Cho and Sean P. Meyn. Licensed under the Creative Commons AttributionNonCommercial License 3.0. Available at http: //econtheory . org.

DOI: $10.3982 /$ TE324 
If there is an unexpected increase in demand, then it will take some time for supply to catch up with demand. However, one might still expect the market price to hover near the marginal production cost as long as the supply side friction is not severe. This reasoning leads to the conclusion that extreme price volatility, or a persistent deviation of the market price away from the marginal cost, is evidence of market failure or the presence of noncompetitive behavior. In this paper we ask whether these conclusions are truly justified.

This research project was motivated in part by events in the California wholesale electricity market between 1998 and 2000, which apparently did not function as envisioned by policy makers. As early as 1998, the ancillary service market repeatedly experienced dramatic price spikes, followed by extended periods of near zero prices, which is considered as early evidence of market dysfunction (Wolak et al. 1998, Fels and Lindh 2001, and Navarro and Shames 2003). This prompted a series of attempts to improve the market design (e.g., Borenstein et al. 2002, Wood et al. 2002, Oren 2002, and Federal Energy Regulatory Commission 2004). The challenge is eloquently described by Borenstein (2001):

The difficulties that have appeared in California and elsewhere are intrinsic to the design of current electricity markets: Demand exhibits virtually no price responsiveness and supply faces strict production constraints and very costly storage. Such a structure will necessarily lead to periods of surplus and of shortage, the latter resulting from both real scarcity of electricity and from sellers exercising market power. Extreme volatility in prices and profits will be the outcome.

The main objective of this paper is to address two fundamental questions. Suppose that observed market prices show repeated upward spikes followed by extended periods in which prices are essentially zero, showing no tendency to converge to the marginal production cost. Suppose in addition that the data show that suppliers extract surplus exceeding what they would obtain if the market price matched the marginal production cost.

First is a positive question. Can a perfectly competitive market with a small amount of friction generate such an outcome? Based on these observations, can we conclude that the market is dysfunctional and some suppliers are exercising market power? Second is a normative question. Are wild fluctuations of market prices harmful for social welfare? Can the market achieve an efficient allocation?

We formulate our questions in terms of a dynamic general equilibrium model in which market demand evolves in continuous time and a consumer realizes a surplus only if the service is delivered. If the service cannot be delivered despite the standing order, the consumer suffers "inconvenience," which is quantified as negative utility. The supplier can provide the service instantaneously up to its capacity at zero marginal cost. The supplier must pay for the cost of capacity. We assume that all agents are price takers, rigorously eliminating any room for market manipulation. The market is subject to supply side friction: Capacity cannot be increased instantaneously, although capacity can be shut down instantaneously without incurring any extra cost (free disposal).

We rigorously analyze the competitive equilibrium and explore its properties. Under the assumption of linear capacity cost and a finite ramping rate on supply, we obtain 
a closed form solution for the equilibrium price functional, which clearly reveals how small supply friction combined with small volatility in demand can result in wild price fluctuations.

Based on these results, we conclude that the marginal production cost is no longer a determining factor for the market clearing price. Moreover, fluctuations of market prices are implied rather than refuted by an efficient market.

Depending upon the initial condition of the economy, it is possible that the supplier extracts a seemingly excessive portion of the gains from trading, while the market remains efficient and free from strategic behavior.

In some markets where demand is unpredictable and the consumer must bear the cost of inconvenience, it is a common practice for the consumer to purchase an extra amount of the good over the instantaneous demand as a precautionary measure. This is known as the reserve. If the consumer cannot purchase extra units for future use since the good is not storable, then the consumer makes a contract with the supplier to maintain extra capacity so that the supplier can offer service immediately in response to a sudden increase in demand. While this extra capacity serves as the reserve in the conventional sense, it is the supplier rather than the consumer who keeps the excess capacity and who is paid for the extra capacity by the consumer.

Theorem 1 contains a complete characterization of the competitive equilibrium outcome, while Theorem 2 describes its welfare properties. The closed form solution for the equilibrium price functional is represented as a function of simple equilibrium state variables, rather than the entire history of outcomes. If the costs are linear, then the equilibrium price at time $t$ can be expressed as a function of the equilibrium reserve $R^{e}(t)$ and demand $D(t)$ via $P^{e}(t)=p^{e}\left(R^{e}(t), D(t)\right)$. The equilibrium price functional is a piecewise constant function of the equilibrium reserve process,

$$
p^{e}\left(r^{e}, d\right)=\left(v+c^{\mathrm{bo}}\right) \mathbb{I}\left\{r^{e}<0\right\},
$$

where $c^{\text {bo }}$ is the marginal cost of inconvenience imposed upon the consumer in case demand is not met and $v$ is the reservation value of the consumer. The sum $c^{\mathrm{bo}}+v$ is, in fact, the maximum price the consumer is willing to pay, often called the choke-up price.

A sample path of the three dimensional process $\left(P^{e}(t), R^{e}(t), D(t)\right)$ is illustrated in Figure 1 for a discrete-time model in which $\mathbf{D}$ is a random walk on $\mathbb{R}^{1}$

The equilibrium price functional has three most surprising features. First, the realized prices fluctuate between the choke-up price and zero, with no tendency to converge to the marginal production cost, regardless of the size of the friction. If the market does not have sufficient reserve $\left(r^{e}<0\right)$, then the market price hits the choke-up price. Alternatively, the market price falls to zero if there is excess capacity $\left(r^{e}>0\right)$. Wild swings of prices arise even if the market is free from any strategic manipulation.

Second, the equilibrium allocation is efficient. The deviation of the market price from the marginal production cost is often suggested as evidence of market dysfunction

\footnotetext{
${ }^{1}$ It can be shown that the optimal reserve process is obtained using a hedging-point policy in this case (Meyn 2007). The hedging-point value $\bar{r}^{*}$ used in this simulation was obtained using the diffusion heuristic of Meyn (2007), which coincides with the value given in Proposition 1 that follows.
} 


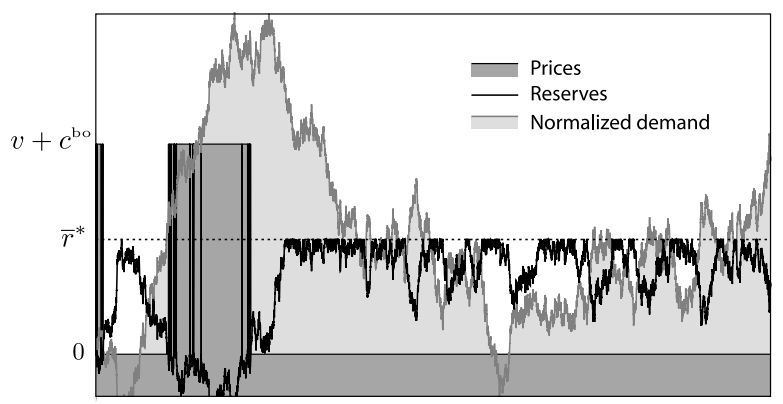

Figure 1. Price dynamics with Brownian demand.

(Fels and Lindh 2001). This observation is accurate in a market without any supply side friction. But in the presence of supply side friction, the supplier has a reason to increase the reserve, even if the spot market price for the reserve is zero, because a large reserve can meet a large demand in the future.

Third, the marginal production cost is irrelevant in determining (1) (or its generalization given in Theorem 1), however large the ramping rate constraint might be, because only two extreme prices will be realized along the sample paths: zero and the choke-up price. However, the link between the market price and the marginal production cost is not completely lost. If the demand evolves according to a Brownian motion, we can characterize the distribution of reserve in an efficient allocation and compute the expected price, which depends on the initial condition of the economy. For sufficiently large initial reserves, the expected discounted average price coincides with the marginal production cost. The expected price increases with decreasing initial reserves (approximating the choke-up price in the limit as normalized initial reserves approach $-\infty$ ). The potentially higher prices mean that a firm can generate a positive profit for an extended period, if not indefinitely.

The dramatic swings of market prices are a consequence of the lack of tradable capacity that can adjust its level of service instantaneously in response to fluctuations in demand. Unless we have a market in which the fast service can fetch a high price, no firm would be willing to provide such a service. Thus, it is not unreasonable to presume that the competitive outcome without a market for the ramping rate should be inefficient.

Our analysis shows otherwise. The spot market for the service perfectly emulates the market for the ramping rate, thus compensating for the absence of the latter to achieve an efficient allocation. A fast ramping rate is valuable only if the society is short on reserve (i.e., $R^{e}(t)<0$ ). Because the service is not storable, the price the consumer is willing to pay on the spot is $v+c^{\text {bo }}$, which is exactly the same price obtained from (1).

This paper examines a market in which the number of active firms is fixed. However, the key insights extend to a market without entry barriers, with some notable differences from the long-run static competitive equilibrium. One can differentiate an inside firm from an outside firm, depending upon the location of its production capacity. One can model entry as a service provided by a firm outside of the market for the consumers 
Theoretical Economics 5 (2010)
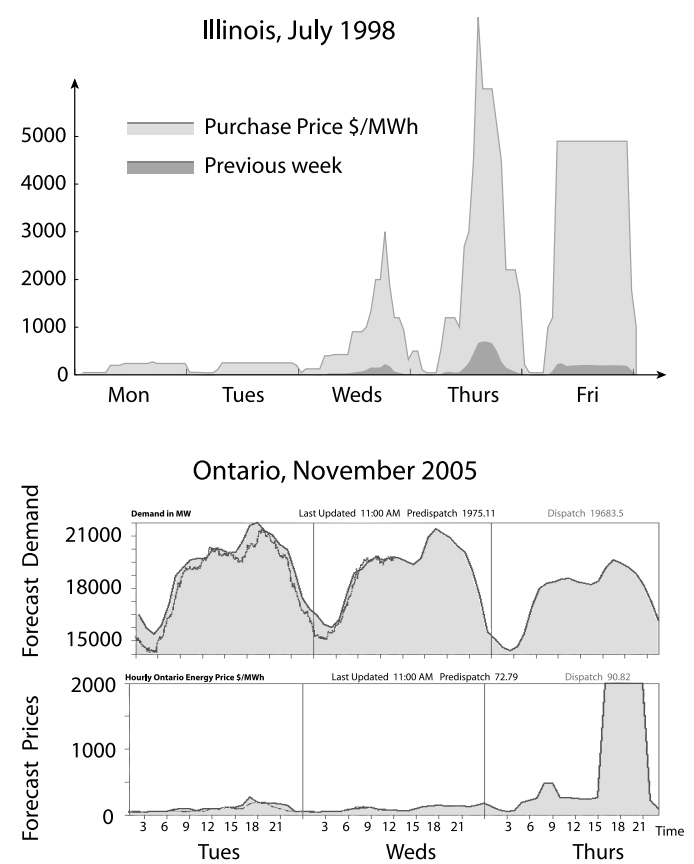
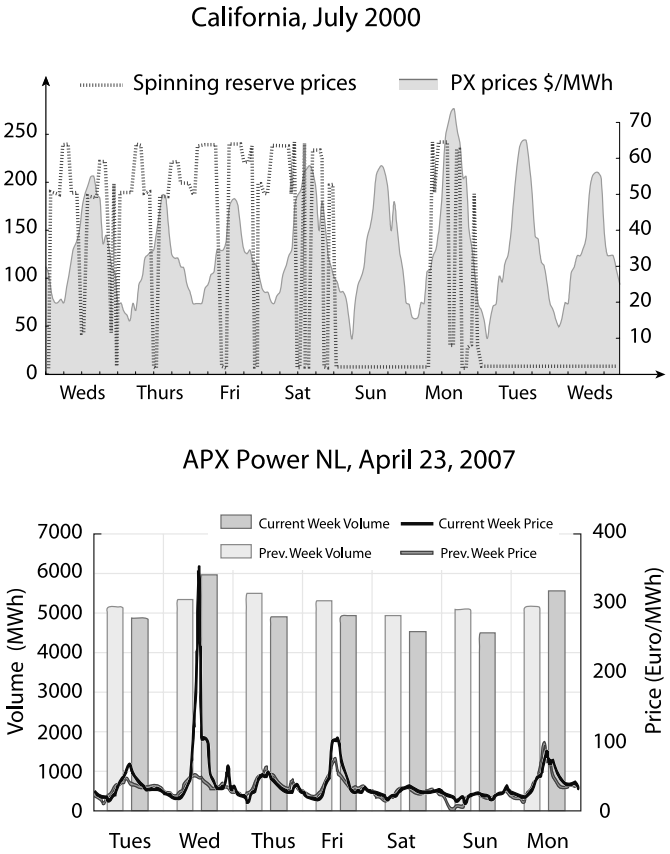

FIgURE 2. Examples of prices in the electric power industry.

inside of the market. Similarly, exit can be formulated as a reduction of service provided by the outside firms. The new service provided by the outside firm helps the market respond to the demand more rapidly, which leads to a lower discounted average market price. If the outside firms can enter and exit instantaneously without any friction, the market price will then coincide with marginal production cost.

The prices obtained from the equilibrium price functional (1) replicate the important qualitative features of the price dynamics shown in Figure 2 for four different wholesale electricity markets in Europe and North America. Note that the prices of power in Ontario and Europe are recent, and the severe volatility shown in the European market is typical behavior that has persisted for several years. However, we do not assert that the recent episodes in California were not caused by strategic behavior of the participantsthere is ample evidence of noncompetitive behavior. Rather, the main result of this paper should serve as a reminder that the insights built around a static analysis can be fragile. The welfare evaluation of dynamic markets with friction requires new analytic tools and a fresh perspective.

In contrast to most existing results based on a static analysis (e.g., Wolfram 1998 and Hortacsu and Puller 2004), Mansur (2008) examines the dynamic decision problem of the generator subject to upward and downward ramping constraints to show that the resulting optimal policy of the price-taking generator may deviate from marginal cost pricing. Our model differs from Mansur (2008) as we obtain the deviation from marginal cost pricing as a consequence of the upward ramping rate constraint alone. Scoring and 
settlement rules are proposed in Chao and Wilson (2002) to implement an efficient allocation in the ancillary service market. Our model suggests that even without an elaborate auction mechanism, the competitive market can internalize the supply side friction to achieve an efficient allocation in a dynamic sense.

Throughout this paper, by capacity, we mean online capacity that can respond to the demand instantaneously. This is distinct from the physical production capacity that determines the maximum supply of service. The physical production capacity is implicitly assumed to be so large that the change of the online capacity is not constrained, so as to ensure that the ramping constraint is the only friction in the economy.

Constraints on the physical production capacity fundamentally affect the market outcome. The nature of this dependency is the topic of the recent paper by Garcia and Stachetti (2008), which considers the investment decision of duopoly firms facing stochastically increasing demand. Short-run friction such as ramping constraint are assumed away, and instead the authors examine long-run production expansion/contraction. Instead of the competitive market, Garcia and Stachetti (2008) explores a dynamic game built upon the duopoly market of Kreps and Scheinkman (1983), in which the firms compete in price in each period subject to the physical production capacity, followed by the investment decision to change the production capacity in the next round. The Bertrand competition between firms producing homogeneous goods does not sufficiently reward a firm with a large market share, and fails to provide incentive for firms to invest efficiently to meet the increasing demand. In the end, the market does not have sufficient reserve capacity and often generates high equilibrium prices. $^{2}$

The management of reserves is very similar to inventory management in manufacturing applications. While the bulk of research has concentrated on joint pricing and inventory control in a single-period model, ${ }^{3}$ dynamic versions of this problem are treated in recent work (Paschalidis and Tsitsiklis 2000, Kleywegt 2001, Monahan et al. 2004, and Bertsimas and de Boer 2005). In some special cases it is found that price is roughly independent of state (Paschalidis and Tsitsiklis 2000), in sharp contrast to what is found in this paper. A two period inventory control problem is introduced in Van Mieghem (1999) to show that under certain conditions, a unique price process exists that can support the efficient allocation; the present paper investigates an infinite-horizon model.

Several recent papers analyze energy markets by applying dynamic programming techniques. Sethi et al. (2005) investigate a stochastic model for an electricity market, while $\mathrm{Wu}$ and Chen (2005) examine dynamic competitive equilibria for a model of the petroleum market. A key conclusion is that the competitive market is efficient and that prices coincide with marginal cost. However, to obtain these conclusions the authors explicitly assume that no hard constraints are active. The finite ramping constraint is often binding in the model considered here, which is shown to generate discontinuities in the market outcomes.

The remainder of the paper is organized as follows. Section 2 formally describes the model. Section 3 contains a characterization of the competitive equilibrium outcome and its welfare properties. The properties of the competitive equilibrium are further analyzed in Section 4 . Section 5 concludes the paper.

\footnotetext{
${ }^{2}$ For a stochastic dynamic treatment of the centralized problem, see Bohn et al. (1984).

${ }^{3}$ See surveys in McGill and van Ryzin (1999), Petruzzi and Dada (1999), and Bertsimas and de Boer (2005).
} 


\section{Model}

We examine a market consisting of a single consumer and supplier who are price takers. These two price-taking agents actually represent an aggregate of a continuum of identical infinitesimally small consumers and suppliers.

\subsection{Demand}

We let $G(t)$ denote the available capacity, $D(t)$ denote the demand, and

$$
R(t)=G(t)-D(t)
$$

denote the reserve at time $t$. Let $\mathbf{G}$ be the process of the available capacity. We often refer to the commodity as the service since it is not storable. Once $g=G(t)$ units of capacity are built, the supplier can deliver up to $g$ units of service freely and instantaneously at time $t$.

It is assumed that consumer demand is perfectly inelastic. Throughout the paper, by demand, we mean a normalized demand obtained by subtracting the forecasted demand from the actual demand in each period. Thus, the (normalized) demand can be negative, if the actual demand is less than its forecast. It is assumed that actual demand at $t=0$ is known, which is normalized to 0 .

For computation it is convenient to consider the Gaussian model

$$
D(t)=N(t)
$$

where $\mathbf{N}$ is a driftless Brownian motion with instantaneous variance $\sigma^{2}$ and $N(0)=0$.

Throughout this paper, we use a bold-faced letter to represent a stochastic process, an uppercase letter to denote a random variable, and a lowercase letter for its realized value. For example, we write $\mathbf{D}=\{D(t) \mid t \geq 0\}$ for the stochastic demand process and let $d \in \mathbb{R}$ denote a possible value of $D(t)$ for a given $t \geq 0$.

\subsection{Supply}

Production is subject to friction, in the sense that the production capacity cannot increase instantaneously: There exists $\zeta \in(0, \infty)$ such that for all $t \geq 0$ and all $t^{\prime}>t$,

$$
\frac{G\left(t^{\prime}\right)-G(t)}{t^{\prime}-t} \leq \zeta
$$

Alternatively, we maintain the assumption of free disposal so that no corresponding lower bound is imposed. ${ }^{4}$ Finally, we assume that $G(t)$ is progressively measurable with respect to the history $\{G(0), D(s) \mid s \leq t\}$.

\footnotetext{
${ }^{4}$ In the power industry, generators are also subject to downward ramping constraints, which prevents a generator from dropping the supply instantaneously. The free disposal assumption actually strengthens the conclusions of this paper: Even if the generator is allowed to drop the supply instantaneously in case of excess supply, our analysis shows that the generator will not do so. Instead, it will let the market price drop to zero.
} 
The production technology of the supplier is subject to a production cost $c(G(t))$ for the production capacity $G(t)$ made available at time $t \geq 0$. The cost is incurred, regardless of the delivery of the goods. We assume that the cost is a linear function of $G(t)$, of the form $c G(t)$ for some constant $c>0$. For each unit of the good delivered, the consumer obtains $v$ units of utility. Thus, the utility of the consumer is $v \min (D(t), G(t))$. Alternatively, if the demand is not met $(R(t)<0)$, the consumer suffers utility loss $c^{\text {bo }} R(t)$ for some $c^{\text {bo }}>0$.

Linearity is imposed on both the production cost of the producer and the inconvenience cost of the consumer so as to obtain the closed form solution for the equilibrium and to highlight the structure of the equilibrium outcome. Many of the key insights of this paper can be obtained for nonlinear, convex cost functions, but this requires more cumbersome notation.

\subsection{Equilibrium and efficiency}

The (spot) price at which the service is traded is denoted $P(t)$ at time $t$. For a given price process $\mathbf{P}$, the payoffs of the supplier and the consumer in period $t$ are defined, respectively, as

$$
\begin{aligned}
\mathcal{W}_{\mathrm{S}}(t) & :=P(t) G(t)-c G(t) \\
\mathcal{W}_{\mathrm{D}}(t) & :=v \min (D(t), G(t))-c^{\mathrm{bo}} \max (0,-R(t))-P(t) G(t) .
\end{aligned}
$$

It is assumed for simplicity that the consumer and the supplier have the same time preference, represented by discount rate $\gamma>0$. The consumer's and the supplier's objective function is the long-run discounted expected profit with discount rate $\gamma$, represented for a given initial reserve $R(0)=r$ by

$$
K_{\mathrm{S}}(r):=\mathrm{E}\left[\int e^{-\gamma t} \mathcal{W}_{\mathrm{S}}(t) d t\right] \quad \text { and } \quad K_{\mathrm{D}}(r):=\mathrm{E}\left[\int e^{-\gamma t} \mathcal{W}_{\mathrm{D}}(t) d t\right]
$$

Whenever the meaning is clear from the context, we suppress the initial condition.

A goal of this paper is to construct price and reserve processes that form a competitive equilibrium.

Definition 1. A competitive equilibrium is a pair of price and supply processes $\left\{\mathbf{P}^{e}, \mathbf{G}^{e}\right\}$ satisfying

$$
\mathbf{G}^{e} \in \underset{\mathbf{G}}{\arg \max }\left[\int e^{-\gamma t} \mathcal{W}_{\mathbf{S}}(t) d t\right]
$$

subject to the ramping constraint (3) and

$$
\mathbf{G}^{e} \in \underset{\mathbf{G}}{\arg \max }\left[\int e^{-\gamma t} \mathcal{W}_{\mathrm{D}}(t) d t\right] .
$$

In this case, $\mathbf{P}^{e}$ is called a competitive equilibrium price process, and we let $\mathbf{R}^{e}=\mathbf{G}^{e}-\mathbf{D}$ denote the equilibrium reserve process. 
The supplier and consumer are each subject to the measurability constraint in their respective optimization problems (5) and (6). The set of feasible strategies for the supplier is subject to the ramping constraint (3), but the consumer's optimization problem is not affected by the ramping constraint in (6). The absence of the ramping constraint in the consumer's optimization problem is critical for our analysis.

We use efficiency as the criterion for evaluating the welfare performance of the market. For an initial condition $r=R(0)$, define

$$
K(r)=\mathrm{E}\left[\int e^{-\gamma t} \mathcal{W}(t) d t\right]
$$

Definition 2. A social planner's problem is

$$
\max _{\mathbf{G}} K(r)
$$

subject to the ramping constraint (3), where

$$
\mathcal{W}(t)=\mathcal{W}_{\mathrm{S}}(t)+\mathcal{W}_{\mathrm{D}}(t)
$$

and its solution $\mathbf{G}^{*}$ is called an efficient allocation.

We assume that the social planner's problem (7) has a solution, which is indeed the case if the demand process evolves according to (2). The proof of Proposition 1 is contained in the Appendix.

Proposition 1. Suppose that $\mathbf{D}$ evolves according to (2) and that all costs are linear. For a given discount rate $\gamma>0$, let $\theta_{+}$denote the positive solution to the quadratic equation

$$
\frac{1}{2} \sigma^{2} \theta^{2}-\zeta \theta-\gamma=0
$$

and define the positive threshold $\bar{r}^{*}$ via

$$
\bar{r}^{*}=\frac{1}{\theta_{+}} \log \left(\frac{c^{\mathrm{bo}}+v}{c}\right)
$$

Then the social planner's problem has a solution and the efficient allocation is characterized as a threshold policy, so that $\mathbf{R}^{*}$ is a reflected Brownian motion on $\left(-\infty, \bar{r}^{*}\right]$ satisfying

$$
\frac{d G^{*}(t)}{d t}=\zeta \quad \text { if } R^{*}(t)<\bar{r}^{*}
$$

Let $\left\{\mathbf{P}^{e}, \mathbf{G}^{e}\right\}$ be a competitive equilibrium. If $\mathbf{G}^{e}$ is an efficient allocation, then we say that the equilibrium is efficient. If every competitive equilibrium is efficient, then we say that the first welfare theorem holds. 


\subsection{Economy without ramping constraint: Benchmark}

Suppose that the supplier can adjust the production capacity instantaneously at each period $(\zeta=\infty)$. Then, in the competitive equilibrium, the production capacity must be such that it can provide the demand at each point of time, and the market price must be precisely the marginal capacity cost (as long as it is less than the choke-up price). In case of linear cost satisfying $c<v+c^{\text {bo }}$, the following identities hold:

$$
P^{e}(t)=c \quad \text { and } \quad G^{e}(t)=D(t), \quad t \geq 0 .
$$

Note that the supplier's optimal strategy is myopic: Produce the service as long as the marginal production cost does not exceed the choke-up price.

As a result, the market outcome of the dynamic model is little more than the repetition of the static outcomes. Since the supplier can produce each unit at the same marginal cost, the market price is equal to $c$ for each $t$. The competitive equilibrium is efficient in an economy without friction. In particular, if the cost function is linear, then the supplier's surplus is 0 and the market clearing price should show no volatility. ${ }^{5}$

If $\zeta<\infty$ is very large, then the economy is "close" to an economy without any friction, because the supplier can respond to the excess demand quickly, if not instantaneously. An important question is whether the market outcome is close to the outcome realized when the supply is frictionless. The answer is anything but expected.

\section{Analysis}

For given initial condition $R(0)=r$, we denote the maximized expectations in (4) by, respectively,

$$
K_{\mathrm{S}}^{*}(r)=\sup \mathrm{E}\left[\int e^{-\gamma t} \mathcal{W}_{\mathrm{S}}(t) d t\right]
$$

and

$$
K_{\mathrm{D}}^{*}(r)=\sup \mathrm{E}\left[\int e^{-\gamma t} \mathcal{W}_{\mathrm{D}}(t) d t\right] .
$$

The price process $\mathbf{P}$ is given, which determines the welfare function $\mathcal{W}_{\mathrm{D}}(t)=$ $w_{\mathrm{D}}(R(t), t)$ with $w_{\mathrm{D}}$ defined in (14). The value function (9) admits the simple upper bound

$$
K_{\mathrm{D}}^{*}(r)=\max _{\mathbf{G}_{\mathrm{D}}} \mathrm{E}\left[\int_{0}^{\infty} e^{-\gamma t} \mathcal{W}_{\mathrm{D}}(t) d t\right] \leq \mathrm{E}\left[\int_{0}^{\infty} \max _{G_{\mathrm{D}}(t)}\left\{e^{-\gamma t} \mathcal{W}_{\mathrm{D}}(t)\right\} d t\right]
$$

We see in Lemma 1 that this upper bound is attained, which implies that the consumer is myopic. Lemma 1 is the key result used in Theorem 1 to prove that the market price is completely determined by the demand side of the market.

\footnotetext{
${ }^{5}$ For a general smooth convex cost function, the equilibrium price volatility should be the same order of magnitude as the demand volatility.
} 
Lemma 1. The solution to the consumer's problem is myopic: For each $t>0$ we have $G_{\mathrm{D}}(t) \in \arg \max \mathcal{W}_{\mathrm{D}}(t)$ and

$$
\mathcal{W}_{\mathrm{D}}(t)=w_{\mathrm{D}}^{*}(t):=\max _{r} w_{\mathrm{D}}(r, t)
$$

Proof. This is a consequence of the assumption that the consumer does not impose rate constraints in the optimization (9). Hence the upper bound in (10) can be realized by enforcing (11) for any $t>0$.

The myopic nature of the consumer's solution implies that the value function is independent of $r$.

LEMмa 2. The value function for the consumer is independent of $r$ :

$$
K_{\mathrm{D}}^{*}(r)=K_{\mathrm{D}}^{*}(0), \quad r \in \mathbb{R} .
$$

Proof. For any initial condition $r=R(0)$, an optimal solution maximizes $R(0+)$ over all initial conditions to achieve

$$
\max _{r^{\prime}} K_{\mathrm{D}}^{*}\left(r^{\prime}\right)
$$

The value of $K_{\mathrm{D}}^{*}(r)$ coincides with this maximum, and is hence independent of $r$.

We can easily calculate the demand correspondence $r_{\mathrm{D}}(p(t))$ of the consumer, where $p(t)$ is the market clearing price at $t$ :

$$
r_{\mathrm{D}}(p(t))= \begin{cases}-\infty & \text { if } p(t)>c^{\mathrm{bo}}+v \\ (-\infty, 0] & \text { if } p(t)=c^{\mathrm{bo}}+v \\ 0 & \text { if } 0<p(t)<c^{\mathrm{bo}}+v \\ {[0, \infty)} & \text { if } p(t)=0 .\end{cases}
$$

Note that if $R^{e}(t)$ is an equilibrium reserve at time $t$, then $\left(R^{e}(t), P^{e}(t)\right)$ must be located in the graph of the demand correspondence where $P^{e}(t)$ is the market clearing price. Hence, if $P^{e}(t)$ is a competitive equilibrium price, inverting (12) gives

$$
P^{e}(t)= \begin{cases}c^{\mathrm{bo}}+v & \text { if } R^{e}(t)<0 \\ {\left[0, c^{\mathrm{bo}}+v\right]} & \text { if } R^{e}(t)=0 \\ 0 & \text { if } R^{e}(t)>0 .\end{cases}
$$

The first main result of this paper characterizes the possible competitive equilibrium price processes.

Theorem 1. Suppose that there is a competitive equilibrium $\left\{\mathbf{P}^{e}, \mathbf{G}^{e}\right\}$. Then the competitive equilibrium price process must satisfy $P^{e}(t)=p^{e}\left(R^{e}(t), D(t)\right)$ for $t \geq 0$ given by (13), where $\mathbf{R}^{e}$ is the reserve process in the competitive equilibrium, and the function $p^{e}: \mathbb{R}^{2} \rightarrow \mathbb{R}_{+}$is (1). 
Proof. Define, for each $t$ and $r$,

$$
\tilde{w}_{\mathrm{D}}(r, t)=v \min (D(t), r+D(t))-c^{\mathrm{bo}} \max (0,-r)-P(t)(r+D(t)) .
$$

Lemma 1 states that the solution to the consumer's optimization problem is myopic: For each $t>0$, we have $\mathcal{W}_{\mathrm{D}}(t)=\max _{r^{\prime}} \tilde{w}_{\mathrm{D}}\left(r^{\prime}, t\right)$. If at some time $t$, the welfare function is differentiable, then we can take derivatives to compute the maximizer in a competitive equilibrium, which gives (1).

It is helpful to recall the nature of the contract that is being traded in this market. If the consumer purchases $g>0$ units of capacity, paying the total sum of $p^{e} g$, then he is entitled to the amount of service up to $g>0$ for no additional cost. The marginal price of the service is 0 , as long as the demand for service is less than the available capacity $g>0$.

To secure $g>0$ units of supply, the consumer has to pay $p^{e} g$, which becomes a sunk cost by the time the capacity produces the service. We can regard the price as a two part tariff that consists of two components: the price for the marginal unit and the price for the fixed component. In this case, the marginal price is set to zero and the fixed component is $p^{e} g$. In this sense, $p^{e}$ is not the price paid for the marginal service, but the price of capacity.

The market price hits the choke-up price $c^{\text {bo }}+v$ whenever demand exceeds the available production capacity $\left(R^{e}(t)<0\right)$. Alternatively, the price falls to zero rather than to the marginal cost whenever $R^{e}(t)>0$. Because the production cost has already been generated, the supplier is willing to provide the service at any positive price, and the market price drops down to zero. Because the commodity is not storable, the supplier cannot withdraw the service from the market for later sales nor can the consumer demand more than what he needs today for future consumption, leaving no room for smoothing the price dynamics.

Next, we prove the existence of a competitive equilibrium and characterize its properties.

The conventional approach to construct the competitive equilibrium is first to calculate the supply schedule and find the intersection with the demand schedule. Instead, we take a detour by proving the next lemma, which will later be combined with Lemma 2 to prove the first welfare theorem and establish the existence of a competitive equilibrium.

Lemma 3. If $\left(\mathbf{R}^{e}, \mathbf{P}^{e}\right)$ is a competitive equilibrium, then the three value functions satisfy, for each $R^{e}(0)=r$,

$$
K^{*}(r)=K_{\mathrm{D}}^{*}(r)+K_{\mathrm{S}}^{*}(r)
$$

Proof. A bound in one direction always holds:

$$
K^{*}(r):=\max _{R} \mathrm{E}\left[\int_{0}^{T} e^{-\gamma t}\left[\mathcal{W}_{\mathrm{D}}(t)+\mathcal{W}_{\mathrm{S}}(t)\right] d t\right]
$$


Theoretical Economics 5 (2010)

$$
\begin{aligned}
& \leq \max _{R_{\mathrm{D}}} \mathrm{E}\left[\int_{0}^{T} e^{-\gamma t} \mathcal{W}_{\mathrm{D}}(t) d t\right]+\max _{R_{\mathrm{S}}} \mathrm{E}\left[\int_{0}^{T} e^{-\gamma t} \mathcal{W}_{\mathrm{S}}(t) d t\right] \\
& =K_{\mathrm{D}}^{*}(r)+K_{\mathrm{S}}^{*}(r) .
\end{aligned}
$$

A bound in the reverse direction is obtained by applying the assumption that a competitive equilibrium $\left(\mathbf{R}^{e}, \mathbf{P}^{e}\right)$ exists:

$$
\begin{aligned}
K_{\mathrm{D}}^{*}(r)+K_{\mathrm{S}}^{*}(r) & =\left.\mathrm{E}\left[\int_{0}^{T} e^{-\gamma t}\left[\mathcal{W}_{\mathrm{D}}(t)+\mathcal{W}_{\mathrm{S}}(t)\right] d t\right]\right|_{R=R^{e}} \\
& \leq \max _{R} \mathrm{E}\left[\int_{0}^{T} e^{-\gamma t}\left[\mathcal{W}_{\mathrm{D}}(t)+\mathcal{W}_{\mathrm{S}}(t)\right] d t\right]=K^{*}(r) .
\end{aligned}
$$

We are ready to prove the first welfare theorem.

\section{Theorem 2. Any competitive equilibrium is efficient.}

Proof. Let $\mathbf{R}^{e}$ denote the reserve process in the competitive equilibrium and let $K(r)$ denote the resulting social welfare. To prove the result, we establish that $K(r)=K^{*}(r)$.

Combining Lemma 3 with Lemma 2 gives

$$
\begin{aligned}
K(r) & =\left.\mathrm{E}\left[\int e^{-\gamma t}\left[w_{\mathrm{D}}^{*}(t)+\mathcal{W}_{\mathrm{S}}(t)\right] d t\right]\right|_{R=R^{e}} \\
& =\left.\max _{R_{\mathrm{S}}} \mathrm{E}\left[\int e^{-\gamma t}\left[w_{\mathrm{D}}^{*}(t)+\mathcal{W}_{\mathrm{S}}(t)\right] d t\right]\right|_{R=R_{\mathrm{S}}} \\
& \geq\left.\max _{R_{\mathrm{S}}} \mathrm{E}\left[\int e^{-\gamma t}\left[\mathcal{W}_{\mathrm{D}}(t)+\mathcal{W}_{\mathrm{S}}(t)\right] d t\right]\right|_{R=R_{\mathrm{S}}}=K^{*}(r),
\end{aligned}
$$

where $w_{\mathrm{D}}^{*}(t)$ is defined in (11). The equality in (15) is implied by the fact that $w_{\mathrm{D}}^{*}(t)$ is independent of $R_{\mathrm{S}}$. The inequality of (16) follows from the fact that the consumer's optimization problem is myopic, so that (11) holds. By definition of $K^{*}(r)$, this bound implies that $K(r)=K^{*}(r)$ as claimed.

It is challenging to calculate a supply schedule because of the ramping constraint appearing in the producer's optimization problem. Yet, we can prove that if $\mathbf{R}^{e}$ solves the social planner's problem (7), then $\mathbf{D}^{e}=\mathbf{R}^{e}+\mathbf{D}$ constitutes a competitive equilibrium together with $\mathbf{P}^{e}$ given by (13).

Proposition 2. Let $\mathbf{G}^{*}$ be the solution of (7). If $\mathbf{P}^{e}$ is given by (13), $\left\{\mathbf{P}^{e}, \mathbf{G}^{*}\right\}$ is a competitive equilibrium.

Proof. Recall that $\mathcal{W}_{\mathrm{S}}(t)=P(t) G(t)-c(G(t))$ and

$$
\mathcal{W}(t)=v \min (D(t), G(t))+c^{\mathrm{bo}} \min (R(t), 0)-c(G(t)) .
$$


If $P(t)=P^{e}(t)$ is given by (13), then the difference of these welfare functions can be expressed as a function of exogenous variables:

$$
\begin{aligned}
\mathcal{W}(t)-\mathcal{W}_{\mathrm{S}}(t) & =(v-P(t)) D(t)+\left(v+c^{\mathrm{bo}}\right) \min (0, R(t))-P(t) R(t) \\
& =(v-P(t)) D(t) .
\end{aligned}
$$

Since $(v-P(t)) D(t)$ is beyond the control by a price-taking producer, then

$$
\mathbf{R}^{*} \in \underset{\mathbf{G}}{\arg \max }\left[\int e^{-\gamma t} \mathcal{W}_{\mathbf{S}}(t) d t\right]
$$

if and only if

$$
\mathbf{R}^{*} \in \underset{\mathbf{G}}{\arg \max } \mathrm{E}\left[\int e^{-\gamma t} \mathcal{W}(t) d t\right]
$$

while both optimization problems are subject to the same ramping constraint. Thus, $\mathbf{G}^{*}$ is the supply schedule induced by $\mathbf{P}^{e}$.

It remains to prove that $\mathbf{R}^{*}=\mathbf{G}^{*}-\mathbf{D}$ solves the consumer optimization problem. Note that if $P^{e}(t)=v+c^{\text {bo }}$, which occurs when $R^{*}(t) \leq 0$, then any $R(t) \leq 0$ maximizes the consumer utility and, in particular, $R^{*}(t) \leq 0$ does. Similarly, if $P^{e}(t)=0$, which occurs when $R^{*}(t) \geq 0$, any $R(t) \geq 0$ maximizes the consumer utility, including $R^{*}(t) \geq 0$. This concludes the proof.

\section{Properties of Competitive EQUilibria}

It is remarkable that the marginal production cost is irrelevant in determining (1), however large the ramping rate constraint $\zeta>0$ might be. The equilibrium price functional (1) reveals that a competitive equilibrium entails extreme price volatility. The efficient equilibrium price can fluctuate between zero and the choke-up price as the market demand fluctuates. Thus, one cannot deduce market failure based on extreme price volatility or persistent deviation from the marginal production cost.

The high market price signals the shortage of the reserves, which prompts the producer to ramp up the supply of service. However, the low price does not necessarily lead to a reduction in supply. If there exist abundant reserves, say $R(t)>r^{*}$, where $r^{*}$ is defined by (8), then the market clearing price is zero and the producer will ramp down the supply quickly. However, if $0<R(t)<r^{*}$, the producer instead ramps up the supply, despite the fact that the market price is zero. The large reserve serves two purposes. First, a large reserve can meet a large demand today. Second, with the presence of the ramping constraint, a large reserve today is useful to meet a potentially large demand tomorrow. If $R(t)<r^{*}$, the benefit from maintaining a large reserve outweighs the cost of providing the additional reserve, and the producer increases supply.

Let us examine how the equilibrium outcome depends on key factors such as the ramping constraint. First, note that the threshold $r^{*}$ decreases as the ramping rate $\zeta>0$ increases. Hence the value of additional reserve decreases as the producer can respond more rapidly to a sudden increase in demand. 
Next we examine the distribution of welfare in the competitive equilibrium by applying Theorem 2 and properties of the efficient allocations summarized in Proposition 1. For this, we recall the representation for $K(r)$ given in (25), based on the exponential random variable $T$. Using the fact that $\bar{r}^{*}-R(T)$ has an exponential distribution combined with the definition of the parameter $\theta^{*}$, we obtain

$$
\mathrm{P}\left\{R(T) \leq 0 \mid R^{e}(0) \geq \bar{r}^{*}\right\}=e^{-\theta^{*} \bar{r}^{*}}=e^{-\log \left(\left(c^{\mathrm{bo}}+v\right) / c\right)}=\frac{c}{c^{\mathrm{bo}}+v} .
$$

Again assuming $R^{e}(0) \geq \bar{r}^{*}$, the average price can be computed as

$$
\begin{aligned}
\mathrm{E}\left[P^{e}(T) \mid R^{e}(0) \geq \bar{r}^{*}\right] & =\left(c^{\mathrm{bo}}+v\right) \mathrm{P}\{R(T) \leq 0\}+0 \mathrm{P}\{R(T)>0\} \\
& =\left(c^{\mathrm{bo}}+v\right) \frac{c}{c^{\mathrm{bo}}+v}+0=c .
\end{aligned}
$$

Consequently, assuming large initial reserves, the discounted average expected price is exactly the marginal production cost of the service in a competitive equilibrium, which is consistent with the conclusion that the outcome is efficient.

Similar arguments show that the expected price is strictly increasing as reserves decrease, for any initial $r<\bar{r}^{*}$, so that $\mathrm{E}\left[P^{e}(T) \mid R^{e}(0)=r\right]>c$ for such $r$. The expected price approaches the maximum value $c^{\text {bo }}+v$ as $r$ tends to negative infinity. As a result, if the economy severely lacks reserve capacity at $t=0$, then the long-run discounted average payoff of the supplier may exceed what he would have obtained in the economy without friction. By the same token, the fact that the distribution is skewed in favor of the supplier does not necessarily imply the presence of market power.

A positive profit induces entry, as the firms outside of the market are willing to provide service to the market. To highlight how the insights of the present analysis can be extended, let us consider a particularly simple model of entry and exit. We differentiate the inside firms from the outside firms according to the location of the production capacity. Entry is the increase of the service provided by the outside firms, and exit is its reduction.

Let us assume that all outside firms and all inside firms share a common production technology, and that the outside firm can enter the market at the rate of $\kappa \geq 0$ at any instant. The model coincides with the one without entry when $\kappa=0$. At the other extreme, when $\kappa=\infty$ the outside firm can respond instantly to an increase in demand. In a certain sense, $\kappa<\infty$ models another form of friction in the economy: the difficulty for outside firms to provide service.

With the service provided by outside firms, the (aggregate) ramping rate is $\zeta(1+\kappa)$. The increment of $\zeta$ comes from the incumbent firms, while $\zeta \kappa$ is provided by the outside firms. The economy is essentially the one with a higher ramping rate, thanks to the outside firms. We can invoke exactly the same analysis to show that the competitive equilibrium with entry is efficient. For the same initial condition on reserve, the discounted average price in the competitive equilibrium is nonincreasing with increasing $\kappa$.

Friction vanishes as $\kappa \rightarrow \infty$, and in the limit the market price coincides with the marginal production cost. However, $\kappa$ is finite in any typical service industry with a large capital investment, so that wild swings in the equilibrium market price can be expected 
(between zero and the choke-up price in this model). Consequently, price volatility cannot be used as evidence of a strategic barrier to entry.

\section{Concluding Remarks}

Although the model is highly stylized, the message is very much relevant to observations of deregulated electricity markets around the world. We have seen that a simple example can replicate the extreme volatility seen in today's power markets. What is remarkable is that extreme volatility is an intrinsic part of the competitive market equilibrium, and the degree of volatility has little to do with the welfare properties of the market outcomes.

Because the marginal pricing rule can be recovered in the competitive market in a weak sense, as described by (17), we can diagnose the presence of noncompetitive behavior by estimating the expected delivery price of the commodity and the expected consumer surplus. While (17) offers a testable implication of the model, we must point out that this is only a mild implication. It is still possible that observed market data can show extremely low consumer surplus, even if the market is efficient. We believe that the diagnosis of market power should be based on a well calibrated dynamic model and its equilibrium outcomes, which includes the collection of feasible price processes. A focus of the dissertation by Ruiz (2008) is the construction of dynamic models that explicitly address ramping rate constraints, based on observed power market data.

We have noted that the existence of a centralized optimal outcome is established in Cho and Meyn (2009) for the model with Brownian demand (2). The analysis allows multiple generators with heterogeneous ramping constraints. The optimal solution is characterized by computable thresholds, much like classical solutions to centralized optimal control in inventory models (Scarf 1963, Meyn 2007). The paper by Chen et al. (2006) contains extensions of the main result of Cho and Meyn (2009) to a network of generators and consumers connected by constrained power lines. Even in the case of Brownian demand, a closed form expression for the social planner's problem is not possible when there are constraints on links. However, it is possible to obtain structural properties of the optimal solution. These results can be applied to understand the competitive benchmark in future market analysis.

\section{Appendix: Proof of Proposition 1}

Proposition 1 states that the optimal reserve process is a reflective Brownian motion (RBM) on $\left(-\infty, \bar{r}^{*}\right]$. To characterize the optimal policy, we first focus on a class of policies under which the reserve process is characterized by an RBM on $(-\infty, \bar{r}]$ for some $\bar{r} \in \mathbb{R}$. Such a policy is called a barrier policy, with barrier $\bar{r}$. To prove Proposition 1 , we

first characterize the optimal barrier $\bar{r}^{*}$ within the class of barrier policies, and we then demonstrate that this policy is optimal among all feasible policies.

\section{A.1 Preliminaries}

The welfare function can be expressed as

$$
\mathcal{W}(t)=(v-c) D(t)+\left(v+c^{\mathrm{bo}}\right) \min (0, R(t))-c R(t) .
$$


On defining the social cost of $R(t)$ as

$$
C(R(t))=c R(t)-\left(v+c^{\mathrm{bo}}\right) \min (0, R(t))= \begin{cases}c R(t) & \text { if } R(t)>0 \\ -\left(v+c^{\mathrm{bo}}-c\right) R(t) & \text { if } R(t) \leq 0,\end{cases}
$$

we express the maximization of expected welfare as the minimization of the expected cost using the identity

$$
-\mathrm{E}[\mathcal{W}(t)]=-\mathrm{E}[(v-c) D(t)]+\mathrm{E}[C(R(t))]=\mathrm{E}[C(R(t))] .
$$

The control variable of the social planner is the sample path of $G(t)$, subject to (3).

For a given $\bar{r}>0$, the barrier policy is defined as a policy under which the reserve process becomes an RBM on $(-\infty, \bar{r}]$ for any initial condition $R(0)$. We shall give an explicit representation for a barrier policy, but first we recall a few key properties of an RBM.

It is convenient to introduce the transformation

$$
Y(t)=\bar{r}-R(t)
$$

so that $\mathbf{Y}$ is an RBM on $[0, \infty)$ under the barrier policy. The evolution of $\mathbf{Y}$ on $\mathbb{R}_{+}$can be expressed as

$$
Y(t)=y-\zeta t+I(t)+D(t), \quad t \geq 0,
$$

where $y=Y(0)=\bar{r}-R(0)$ is the initial condition. The process I is known as the idleness (or reflection) process. The idleness process is nondecreasing, with $I(0)=0$, and satisfies

$$
\int_{0}^{\infty} Y(t) d I(t)=0
$$

This makes precise the notion that $I(t)$ can increase only when $Y(t)=0$ (equivalently, when $R(t)=\bar{r})$.

By an exponential distribution with parameter $\theta^{*}>0$, we mean the probability distribution supported on $\mathbb{R}_{+}$, with density given by

$$
p_{Y}(y)=\theta^{*} e^{-\theta^{*} y}, \quad y \geq 0 .
$$

It is known that the RBM has a stationary distribution of this form.

Proposition 3. The reflected Brownian motion (18) has a unique stationary distribution. It is exponential with parameter $\theta^{*}$, where

$$
\theta^{*}=\frac{2 \zeta}{\sigma^{2}}
$$

For the proof, see Harrison and Williams (1987).

These expressions can be mapped to the reserve process, providing an explicit construction of a barrier policy. Define $I(t)$ as the gap between the maximum rate of production and the actual generation:

$$
I(t)=r+\zeta t-G(t) .
$$


This definition of $\mathbf{I}$ is consistent with (18). Since $r+\zeta t$ is exogenous, we can treat $I(t)$ as a control instead of $G(t)$. Since $R(t)=G(t)-D(t)$, we can write

$$
R(t)=r+\zeta t-I(t)-D(t), \quad t \geq 0,
$$

where $r=R(0)$.

We claim that the barrier policy can be expressed in terms of the observed demand process as

$$
I(t)=\max _{0 \leq \tau \leq t} \max (0,-(\bar{r}-r)+\zeta \tau-D(\tau)), \quad t \geq 0 .
$$

It is clear from this representation that $\mathbf{I}$ is nondecreasing and nonnegative. Moreover, under the assumption that $r \leq \bar{r}$, it follows that $I(0)=0$.

To verify that (21) is indeed a barrier policy, we recall the standard representation of an RBM via the Skorokhod map (cf. Chapter 3 of Meyn 2007). Assume that a barrier policy has been applied, so that $Y(t)=\bar{r}-R(t)$ is an RBM on $[0, \infty)$. The free process describes how $Y(t)$ would have evolved without reflection, that is,

$$
F(t):=(\bar{r}-r)-\zeta t+D(t), \quad t \geq 0 .
$$

Note that $\mathbf{F}$ is a Brownian motion with drift $-\zeta$. The Skorokhod map representation of the RBM $\mathbf{Y}$ is given by

$$
Y(t)=\max _{0 \leq \tau \leq t} \max (F(t), F(t)-F(\tau)), \quad t \geq 0 .
$$

Comparing (18) and (22), we conclude that the idleness process is given by

$$
\begin{aligned}
I(t) & =Y(t)-F(t) \\
& =\max _{0 \leq \tau \leq t} \max (0,-F(\tau)), \quad t \geq 0,
\end{aligned}
$$

which is exactly (21). This proves that (21) describes the barrier policy with barrier $\bar{r}$.

Proposition 3 implies that the stationary distribution of $R(t)$ under a barrier policy is the shifted exponential

$$
p_{R}(r)= \begin{cases}\theta^{*} e^{-\theta^{*}(\bar{r}-r)} & \text { if } r \leq \bar{r} \\ 0 & \text { if } r>\bar{r}\end{cases}
$$

\section{A.2 Without discounting}

It is instructive to apply Proposition 3 to characterize the optimal policy for the case without discounting. The objective function of the social planner is the average cost

$$
\mathcal{C}_{0}(\bar{r})=\limsup _{T \rightarrow \infty} \frac{1}{T} \int_{0}^{T} \mathrm{E}[C(R(t))] d t .
$$


If $R(0)$ is distributed according to the stationary distribution, then

$$
\begin{aligned}
\mathcal{C}_{0}(\bar{r}) & =\mathrm{E}[C(R(T))] \\
& =\int_{-\infty}^{0}\left(v+c^{\mathrm{bo}}-c\right)|x| \theta^{*} e^{\theta^{*}(x-\bar{r})} d x+\int_{0}^{\bar{r}} c x \theta^{*} e^{\theta^{*}(x-\bar{r})} d x,
\end{aligned}
$$

where the first equality holds for any $T$ by stationarity, and the second follows from the Law of Large Numbers combined with (23).

On writing the average cost as the convolution,

$$
\mathcal{C}_{0}(\bar{r})=\int_{0}^{\infty} C(-(\bar{r}-y)) p_{Y}(y) d y,
$$

we see that $\mathcal{C}_{0}(\bar{r})$ is convex and differentiable as a function of $\bar{r}$. We obtain a formula for the value of $\bar{r}$ minimizing $\mathcal{C}_{0}(\bar{r})$ by setting the derivative of the right hand side of (24) equal to zero:

$$
\bar{r}^{*}=\frac{1}{\theta^{*}} \log \left(\frac{v+c^{\mathrm{bo}}}{c}\right) .
$$

Section 7 of Wein (1992) proves that the barrier policy using $\bar{r}^{*}$ is, in fact, an optimal policy among all feasible policies. The proof can be obtained by appealing to the convexity of the relative value function that arises in the associated average-cost dynamic programming equations. In the next section, the value function is constructed under the discounted cost criterion, and we find that it is convex as a function of the initial condition of $\mathbf{R}$.

\section{A.3 With discounting}

Let us denote the discount factor as $e^{-\gamma}$. We initially restrict analysis to barrier policies, as represented in (21). On establishing convexity of the value function, we can conclude as in Section 7 of Wein (1992) that the optimal barrier policy is, in fact, optimal over all policies.

A critical bridge between this problem and the average-cost problem is the interpretation of the discount term $e^{-\gamma t}$ as an unnormalized exponential density. We let $T$ denote an exponential random variable with parameter $\gamma$, independent of the demand process. For a given barrier policy with barrier $\bar{r}$ and a given initial condition $r=R(0)$, the resulting discounted cost can be expressed as

$$
\mathcal{C}_{\gamma}(r ; \bar{r})=\mathrm{E}\left[\int_{0}^{\infty} e^{-\gamma t} C(R(t)) d t \mid R(0)=r\right]=\gamma^{-1} \mathrm{E}[C(R(T)) \mid R(0)=r] .
$$

Note that the only substantive difference between (25) and (24) is that $T$ in (25) is a specially constructed random time, while in (25) the time $T$ is deterministic and the process $\mathbf{R}$ is assumed to be stationary. To compute the optimal policy under discounting, we need only to know the distribution of $R(T)$ when $T$ is an exponentially distributed random variable with parameter $\gamma>0$. We find that its form is similar to (23) when $R(0)=\bar{r}$. 
Proposition 4. Suppose that a barrier policy is applied and that $\mathbf{R}$ is initialized with $R(0)=\bar{r}$. Suppose that $T$ is an exponential random variable with parameter $\gamma$, independent of $\mathbf{D}$. Then the random variable $Y(T)=\bar{r}-R(T)$ has an exponential distribution whose parameter $\theta_{+}$is the positive root of the quadratic equation

$$
\frac{1}{2} \sigma^{2} \theta^{2}-\delta \theta-\gamma=0
$$

Note that as $\gamma \downarrow 0$, we have $\theta_{+} \rightarrow \theta^{*}$, which is the parameter for the exponential distribution that defines the steady-state distribution of $Y(t)$.

Proof of Proposition 4. To prove the proposition, it suffices to compute the moment generating function of $Y(T)$ and to show that for $\vartheta \leq 0$ it coincides with the moment generating function of an exponential random variable with parameter $\theta_{+}$:

$$
\mathrm{E}\left[e^{\vartheta Y(T)} \mid Y(0)=0\right]=\frac{\theta_{+}}{\theta_{+}-\vartheta} .
$$

To this end, we construct solutions to dynamic programming equations for the RBM. The differential generator for $\mathbf{Y}$ is defined by

$$
\mathcal{D} h=-\zeta h^{\prime}+\frac{1}{2} \sigma^{2} h^{\prime \prime}
$$

Lemma 4 provides a dynamic programming equation for the scaled moment generating function for an arbitrary initial condition, that is,

$$
h(y)=\gamma^{-1} \mathrm{E}\left[c_{\vartheta}(Y(T)) \mid Y(0)=y\right],
$$

where $T$ is distributed according to the exponential distribution with parameter $\gamma$. The derivative condition $h^{\prime}(0)=0$ in Lemma 4 is a special case of the boundary condition imposed in Theorem 2 of Harrison and Reiman (1981), and equation (30) of Harrison and Reiman (1981) is similar to the conclusion of the lemma.

Lemma 4. Let $c_{\vartheta}(y)=e^{\vartheta y}$. Suppose that $h: \mathbb{R}_{+} \rightarrow \mathbb{R}$ is a twice continuously differentiable function for which $h$ and $h^{\prime}$ are bounded, and also satisfying

$$
\begin{aligned}
\mathcal{D} h & =\gamma h-c_{\vartheta} \\
h^{\prime}(0) & =0 .
\end{aligned}
$$

Then h coincides with (27). Equivalently,

$$
h(y)=\mathrm{E}\left[\int_{0}^{\infty} e^{-\gamma \tau} c_{\vartheta}(Y(\tau)) d \tau \mid Y(0)=y\right] .
$$

Proof. Define

$$
g(y, t)=e^{-\gamma t} h(y) \quad \text { and } \quad G(t)=g(Y(t), t) .
$$


An application of Itô's formula gives

$$
\begin{aligned}
d G(t)= & g_{t}(Y(t), t) d t+g_{y}(Y(t), t) d Y(t)+\frac{1}{2} \sigma^{2} g_{y y}(Y(t), t) d t \\
= & \left(-\gamma e^{-\gamma t} h(Y(t))+e^{-\gamma t}\left[-\zeta h^{\prime}(Y(t))+\frac{1}{2} \sigma^{2} h^{\prime \prime}(Y(t))\right]\right) d t \\
& \quad+e^{-\gamma t} h^{\prime}(Y(t)) d I(t)+e^{-\gamma t} h^{\prime}(Y(t)) d D(t) \\
= & (-\gamma h(Y(t))+\mathcal{D} h(Y(t))) e^{-\gamma t} d t+e^{-\gamma t} h^{\prime}(Y(t)) d I(t)+e^{-\gamma t} h^{\prime}(Y(t)) d D(t) .
\end{aligned}
$$

By the dynamic programming equation (28), we have

$$
-\gamma h(Y(t))+\mathcal{D} h(Y(t))=-c_{\vartheta}(Y(t)) .
$$

The property (29) combined with (19) implies

$$
h^{\prime}(Y(t)) d I(t)=0 .
$$

Thus, we have

$$
d G(t)=-e^{-\gamma t} c_{\vartheta}(Y(t))+e^{-\gamma t} h^{\prime}(Y(t)) d D(t),
$$

which is equivalent to the integral equation

$e^{-\gamma t} h(Y(t))-h(Y(0))=G(t)-G(0)=-\int_{0}^{t} e^{-\gamma \tau} c_{\vartheta}(Y(\tau)) d \tau+\int_{0}^{t} e^{-\gamma \tau} h^{\prime}(Y(\tau)) d D(\tau)$.

Since $h$ and $h^{\prime}$ are bounded, we can take expectations of each side and let $t \rightarrow \infty$ to obtain the desired formula:

$$
h(y)=\mathrm{E}\left[\int_{0}^{\infty} e^{-\gamma r} c_{\vartheta}(Y(\tau)) d \tau \mid Y(0)=y\right] .
$$

Next we construct a solution to (28) and (29). Since $-2 \gamma / \sigma^{2}<0$, the quadratic equation (26) has two real roots: the parameter $\theta_{+}>0$ defined in Proposition 4 and a second root $\theta_{-}<0$. That is, we can write

$$
\vartheta^{2}-\frac{2 \zeta}{\sigma^{2}} \vartheta-\frac{2 \gamma}{\sigma^{2}}=\left(\vartheta-\theta_{-}\right)\left(\vartheta-\theta_{+}\right) .
$$

Moreover, a small amount of algebra shows that

$$
\theta_{+} \theta_{-}=-\frac{2 \gamma}{\sigma^{2}}<0
$$

We now show that a solution to (28) and (29) is

$$
h(y)=\kappa\left(e^{\vartheta y}-\frac{\vartheta}{\theta_{-}} e^{\theta_{-} y}\right), \quad y \geq 0,
$$

where $\kappa>0$ is a constant to be determined. Clearly, (29) holds. To obtain (28), we require

$$
\kappa\left(\left(-\zeta \vartheta+\frac{1}{2} \sigma^{2} \vartheta^{2}\right) e^{\vartheta y}-\left(-\zeta \vartheta+\frac{1}{2} \sigma^{2} \vartheta \theta_{-}\right) e^{\theta_{-} y}\right)=\gamma h(y)-e^{\vartheta y}, \quad y \geq 0 .
$$


After arranging the terms, this becomes

$$
\left(\kappa\left[\gamma+\zeta \vartheta-\frac{1}{2} \sigma^{2} \vartheta^{2}\right]-1\right) e^{\vartheta y}-\frac{\vartheta \kappa}{\theta_{-}}\left[\gamma+\zeta \theta_{-}-\frac{1}{2} \sigma^{2} \theta_{-}^{2}\right] e^{\theta_{-} y}=0 .
$$

Since $\theta_{-}$is a solution of (26), we have

$$
\gamma+\zeta \theta_{-}-\frac{1}{2} \sigma^{2} \theta_{-}^{2}=0 .
$$

Therefore, to solve (28), the constant $\kappa$ should be selected to make the first term equal to 0 :

$$
\kappa=\left(\gamma+\zeta \vartheta-\frac{1}{2} \sigma^{2} \vartheta^{2}\right)^{-1} .
$$

The final step is to evaluate $\gamma h(0)$ to show that it coincides with the moment generating function of the exponential distribution with parameter $\theta_{+}$:

$$
\gamma h(0)=\frac{\theta_{+}}{\theta_{+}-\vartheta} .
$$

We obtain the desired formula by substituting the expression for $\kappa$ in (32) into the definition of $h$ and applying the identities (30) and (31):

$$
\begin{aligned}
\gamma h(0) & =\gamma \kappa\left(1-\frac{\vartheta}{\theta_{-}}\right)=\frac{\gamma\left(\theta_{-}-\vartheta\right)}{\theta_{-}\left(\gamma+\zeta \vartheta-\frac{1}{2} \sigma^{2} \vartheta^{2}\right)} \\
& =\frac{\gamma\left(\theta_{-}-\vartheta\right)}{\left[-\frac{1}{2} \sigma^{2} \theta_{-}\right]\left(\theta_{-}-\vartheta\right)\left(\theta_{+}-\vartheta\right)}=\left[-\frac{2 \gamma}{\sigma^{2} \theta_{-}}\right]\left[\frac{1}{\theta_{+}-\vartheta}\right]=\frac{\theta_{+}}{\theta_{+}-\vartheta} .
\end{aligned}
$$

The next step in the proof of Proposition 1 is the derivation of the formula (8). This follows exactly the same steps as in the case without discounting, after we replace $\theta^{*}$ by $\theta_{+}$.

These arguments demonstrate only that (8) induces a barrier policy that is optimal among all barrier policies, subject to the constraint that the reserve process is initialized with $R(0)=\bar{r}^{*}$. The final step in the proof of Proposition 1 is to remove these restrictions: We must generalize to arbitrary initial conditions and demonstrate that the optimal policy is indeed a barrier policy. For this, we apply dynamic programming arguments. Combining (20) and the ramping constraint (3), we regard

$$
\mathcal{I}=\{\mathbf{I} \mid \mathbf{I} \text { is adapted to } \mathbf{G} \text {, and nondecreasing, with } I(0)=0\}
$$

as the set of all feasible controls. Let $H^{*}: \mathbb{R} \rightarrow \mathbb{R}$ denote the discounted value function, defined consistently with (4):

$$
H^{*}(r):=\inf _{\mathcal{I}}\left[\int_{0}^{\infty} e^{-\gamma t} C(R(t)) d t \mid R(0)=r\right], \quad r \in \mathbb{R} .
$$

Following the proof of Proposition 9.8.1 in Meyn (2007), we can show that $H^{*}(r)$ is a convex nonincreasing function of $r$. In fact, $H^{*}(r)$ can be computed explicitly: Following the same steps as in the proof of Proposition 3.4.13 in Meyn (2007), we obtain the 
following representation. The value function is constant on $\left[\bar{r}^{*}, \infty\right)$ and, for $r \leq \bar{r}^{*}$,

$$
H^{*}(r)=\gamma^{-1} C(r)+\gamma^{-2} C^{\prime}(r)+ \begin{cases}a_{-} e^{-\theta_{-} r} & \text { if } r<0 \\ b_{-} e^{-\theta_{-} r}+b_{+} e^{-\theta_{+} r} & \text { if } 0 \leq r \leq \bar{r}^{*},\end{cases}
$$

where $\left(\theta_{+}, \theta_{-}\right)$satisfy (30). The derivative $C^{\prime}$ is defined as the piecewise constant function

$$
C^{\prime}(r)= \begin{cases}-\left(v+c^{\text {bo }}-c\right) & \text { if } r<0 \\ c & \text { if } r \geq 0 .\end{cases}
$$

The three constants $\left(a_{-}, b_{-}, b_{+}\right)$are specified by the three constraints

$$
H^{*}(0+)=H^{*}(0-), \quad \frac{d}{d r} H^{*}(0+)=\frac{d}{d r} H^{*}(0-), \quad \frac{d}{d r} H^{*}\left(\bar{r}^{*}\right)=0 .
$$

This function solves the dynamic programming equation for this optimal control problem, as described in Atar and Budhiraja (2006):

$$
\mathcal{D} H^{*}(r)=-c(r)+\gamma H^{*}(r), \quad r<\bar{r}^{*}, \quad \frac{d}{d r} H^{*}\left(\bar{r}^{*}\right)=0 .
$$

It follows from Theorem 2.1 of Atar and Budhiraja (2006) that the associated barrier policy is optimal over all policies for any initial condition.

\section{REFERENCES}

Atar, Rami and Amarjit Budhiraja (2006), "Singular control with state constraints on unbounded domain.” Annals of Probability, 34, 1864-1909. [237]

Bertsimas, Dimitris and Sanne de Boer (2005), "Dynamic pricing and inventory control for multiple products." Journal of Revenue and Pricing Management, 3, 303-319. [220]

Bohn, Roger E., Michael C. Caramanis, and Fred C. Schweppe (1984), "Optimal pricing in electrical networks over space and time.” RAND Journal of Economics, 15, 360-376. [220]

Borenstein, Severin (2001), "The trouble with electricity markets and California's electricity restructuring disaster." Journal of Economic Perspectives, 16, 191-211. [216]

Borenstein, Severin, James B. Bushnell, and Frank A. Wolak (2002), "Measuring market inefficiencies in California's restructured wholesale electricity market." American Economic Review, 92, 1376-1405. [216]

Chao, Hung-Po and Robert Wilson (2002), "Multi-dimensional procurement auctions for power reserves: Robust incentive-compatible scoring and settlement rules." Journal of Regulatory Economics, 22, 161-183. [220]

Chen, Mike, In-Koo Cho, and Sean P. Meyn (2006), "Reliability by design in distributed power transmission networks.” Automatica, 42, 1267-1281. [230]

Cho, In-Koo and Sean P. Meyn (2009), "A dynamic newsboy model for optimal reserve management in electricity markets." Unpublished paper, University of Illinois, UrbanaChampaign. [230] 
Dasgupta, Prithviraj, Louise E. Moser, and P. Michael Melliar-Smith (2005), "Dynamic pricing for time-limited goods in a supplier-driven electronic marketplace." Electronic Commerce Research, 5, 267-292. [215]

Federal Energy Regulatory Commission (2004), "2004 state of the markets report: An assessment of energy markets in the United States in 2004.” Docket MO05-4-000, Federal Energy Regulatory Commission. [216]

Fels, Nicholas W. and Frank R. Lindh (2001), "Lessons from the California "apocalypse:” Jurisdiction over electric utilities.” Energy Law Journal, 22, 1-40. [216, 218]

Garcia, Alfredo and Ennio Stachetti (2008), "Investment dynamics in electricity markets." Unpublished paper, Department of Systems and Information Engineering, University of Virginia. [220]

Harrison, J. M. and R. J. Williams (1987), "Multidimensional reflected Brownian motions having exponential stationary distributions." Annals of Probability, 15, 115-137. [231]

Harrison, J. Michael and Martin I. Reiman (1981), "Reflected Brownian motion on an orthant." Annals of Probability, 9, 302-308. [234]

Hortacsu, Ali and Steven L. Puller (2004), "Testing strategic models of firm behavior in restructured electricity markets: A case study of ercot." Working Paper 125, Center for the Study of Energy Markets. [219]

Kleywegt, A. J. (2001), "An optimal control problem of dynamic pricing." School of Industrial and Systems Engineering, Georgia Institute of Technology. [220]

Kreps, David M. and José A. Scheinkman (1983), "Quantity precommitment and Bertrand competition yield Cournot outcomes.” Bell Journal of Economics, 14, 326-337. [220]

Mansur, Erin T. (2008), "Measuring welfare in restructured electricity markets." Review of Economics and Statistics, 90, 369-386. [219]

McGill, Jeffrey I. and Garrett J. van Ryzin (1999), "Revenue management: Research overview and prospects.” Transportation Science, 33, 233-256. [220]

Meyn, Sean P. (2007), Control Techniques for Complex Networks. Cambridge University Press, Cambridge. [217, 230, 232, 236]

Monahan, George E., Nicholas C. Petruzzi, and Wen Zhao (2004), "The dynamic pricing problem from a newsvendor's perspective." Manufacturing and Service Operations Management, 6, 73-91. [220]

Navarro, Peter and Michael Shames (2003), "Aftershocks—and essential lessons—from the California electricity debacle." Electricity Journal, 16, 24-30. [216]

Oren, Shmuel S. (2002), "Comments in FERC docket no. RM01-12-000: The FGR vs. FTR debate: Facts and misconceptions.” Unpublished paper, Power Systems Engineering Research Center, University of Wisconsin. [216] 
Paschalidis, Ioannis C. and John N. Tsitsiklis (2000), "Congestion-dependent pricing of network services.” IEEE/ACM Transactions on Networking, 8, 171-184. [220]

Petruzzi, Nicholas C. and Maqbool Dada (1999), "Pricing and the newsvendor problem: A review with extensions.” Operations Research, 47, 183-194. [220]

Ruiz, Pablo Ariel (2008), Reserve Valuation in Electric Power Systems. Ph.D. thesis. University of Illinois, Urbana-Champaign. [230]

Scarf, H. E. (1963), "A survey of analytic techniques in inventory theory." In Multistage Inventory Models and Techniques, 185-225, Stanford University Press, Stanford, California. [230]

Sethi, Suresh P., Yan Houmin, J. H. Z. Yan, and Zhang Hanqin (2005), "An analysis of staged purchases in deregulated time-sequential electricity markets." Journal of Industrial and Management Optimization, 1, 443-463. [220]

Van Mieghem, Jan A. (1999), "Coordinating investment, production, and subcontracting.” Management Science, 45, 954-971. [220]

Wein, Lawrence M. (1992), "Dynamic scheduling of a multiclass make-to-stock queue.” Operations Research, 40, 724-735. [233]

Wolak, Frank, Robert Nordhaus, and Carl Shapiro (1998), "Preliminary report on the operation of the ancillary services markets of the California Independent System Operator (ISO)." Unpublished paper, California Independent System Operator. [216]

Wolfram, Catherine D. (1998), "Strategic bidding in a multiunit auction: An empirical analysis of bids to supply electricity in England and wales." RAND Journal of Economics, 29, 703-725. [219]

Wood, Pat, William L. Massey, Linda Breathitt, and Nora Mead Brownell (2002), "Order on a standards development organization for the wholesale electric industry." Docket RM01-12-000, Federal Energy Regulatory Commission. [216]

Wu, Owen Q. and Hong Chen (2005), “Optimal control and competitive equilibrium of production-inventory systems with application to the petroleum refining industry." Unpublished paper, McCombs School of Business, University of Texas, Austin. [220] 